\title{
RANKING DE EVIDENCIAÇÃO DOS IMPACTOS AMBIENTAIS DAS EMPRESAS BRASILEIRAS LISTADAS NO IBrX-100 DA BM\&FBOVESPA - UMA ANÁLISE USANDO O MÉTODO MULTICRITÉRIO TOPSIS
}

\author{
Larissa Degenhart \\ Universidade Regional de Blumenau - FURB, SC, Brasil \\ lari_ipo@hotmail.com \\ Fabricia Silva da Rosa \\ Universidade Federal de Santa Catarina - UFSC, SC, Brasil \\ fabriciasrosa@hotmail.com \\ Nelson Hein \\ Universidade Regional de Blumenau - FURB, SC, Brasil \\ $\underline{\text { hein@furb.br }}$ \\ Mara Vogt \\ Universidade Regional de Blumenau - FURB, SC, Brasil \\ maravogtcco@gmail.com
}

\begin{abstract}
RESUMO
O objetivo deste estudo foi verificar o ranking de evidenciação dos impactos ambientais das empresas brasileiras listadas no IBrX-100 da BM\&FBovespa, utilizando o modelo da entropia e método TOPSIS para a elaboração dos rankings. Trata-se de um estudo descritivo, documental e quantitativo. Os resultados evidenciaram que houve alternância das posições, visto que as empresas que obtiveram as primeiras colocações no ranking geral, ocuparam posições intermediárias nos rankings sobre os aspectos ambientais emissões, efluentes, resíduos, produtos/serviços e transporte. Conclui-se que é importante divulgar informações ambientais, visto que os Relatórios de Sustentabilidade (RS) e Relatórios Anuais (RA) possibilitam que a empresa aprofunde o nível de evidenciação ambiental, obtendo diversos benefícios, como também a sociedade e o meio ambiente.
\end{abstract}

Palavras-chave: Evidenciação Ambiental - Ranking - Empresas brasileiras.

\begin{abstract}
The objective of this study was to investigate the disclosure of ranking the environmental impacts of Brazilian companies listed on the IBrX-100 BM\&FBovespa, using the model of entropy and TOPSIS method for the preparation of rankings. This is a descriptive, document and quantitative study. The results showed that there was alternation of positions, since the companies that scored the first places in the overall ranking, occupied intermediate positions in the rankings on the environmental aspects emissions, effluents, waste, products/services and transport. In conclusion, it is important to disclose environmental information, as the Sustainability Reporting (RS) and Annual Reports (RA) enable the company to deepen the level of environmental disclosure, obtaining many benefits, but also to society and the environment.
\end{abstract}

Keywords: Environmental Disclosure - Ranking - Brazilian companies. 


\section{INTRODUÇÃO}

$\mathrm{Na}$ literatura diversos temas vêm sendo abordados, porém, uma área de interesse ao longo dos anos têm sido as tentativas de explicar a evidenciação ambiental das empresas (GRAY et al., 2001). Rahaman, Lawrence e Roper (2004) salientam que a literatura referente à divulgação ambiental, tem buscado fornecer explicações para as mais diversas decisões corporativas, com vistas a divulgar informações ambientais nos relatórios.

Park e Brorson (2005) abordam que as empresas em todo o mundo, estão preocupadas em evidenciar informações ambientais, por meio dos relatórios, para as partes interessadas e a sociedade. Para tanto, várias empresas, começaram a incluir questões ambientais em seus relatórios ambientais, que aos poucos se converteram em RS. Deste modo, muitas empresas já perceberam os benefícios relacionados a uma maior transparência do desempenho ambiental.

Segundo Bowrin (2013), a tendência das empresas divulgarem voluntariamente informações ambientais, têm sido de grande interesse para os pesquisadores na área de contabilidade nas últimas três décadas. A comunidade científica conforme Rosa et al. (2013), reconhece a importância da avaliação do nível da informação ambiental divulgada pelas empresas, com o intuito de contribuir para o desenvolvimento sustentável, desenvolvendo pesquisas sobre as informações ambientais, que visam a construção de instrumentos que possibilitam apoiar as decisões tomadas sobre a evidenciação ambiental.

Nesse sentido, Boff (2007) salienta que a evidenciação ambiental inspira o desenvolvimento de estudos, pelo fato das empresas estarem cada vez mais pressionadas pela sociedade em divulgarem a sua preocupação com o meio ambiente. Para tanto, necessitam demonstrar que participam da preservação ambiental e valorização da sociedade. Assim, a evidenciação ambiental é necessária para este processo, pois as empresas conseguem informar os resultados obtidos dos programas implantados sobre a questão ambiental.

Diante do contexto exposto, apresenta-se a questão problema que norteia este estudo: Qual o ranking de evidenciação dos impactos ambientais das empresas brasileiras listadas no IBrX-100 da BM\&FBovespa? Deste modo, o objetivo do estudo é verificar o ranking de evidenciação dos impactos ambientais das empresas brasileiras listadas no IBrX-100 da BM\&FBovespa.

O estudo justifica-se, pois segundo Albertini (2013), nos últimos 15 anos, as empresas têm aumentado a divulgação ambiental em resposta às pressões institucionais. Essa demanda da sociedade para uma maior preservação ambiental pelas empresas, juntamente com o aumento da regulação ambiental, faz com que estas desenvolvam e participem de programas de prevenção e controle da poluição.

Justifica-se ainda, pois conforme Rosa et al. (2014), a transparência ambiental das empresas ganha destaque, pois é considerada uma resposta às demandas sociais que se referem ao consumo dos recursos naturais e impactos ambientais, que são causados pelas atividades das empresas, o que ocasiona reflexos no clima, biodiversidade e saúde humana. Destaca-se a importância da evidenciação ambiental, visto que auxilia na tomada de decisões das partes interessadas, dentre outros benefícios para a empresa, sociedade e meio ambiente.

\section{EVIDENCIAÇÃO AMBIENTAL}

O desafio crescente da preservação ambiental tem forçado as empresas a alterarem sua estrutura operacional e melhorarem a divulgação de suas políticas e ações ambientais, pois nas últimas décadas as variáveis ambientais representam uma vantagem competitiva. As empresas devem passar a se preocupar com a preservação ambiental e sua restauração, pois a consciência ambiental está desenvolvendo novas oportunidades de negócios, fazendo com que as empresas alcancem seus objetivos de gestão estratégica (TRIERWEILLER et al., 2012). 
A evidenciação ambiental é considerada um processo por meio do qual as empresas divulgam o seu impacto sobre o meio ambiente. A forma e a integridade com que as empresas fazem esse processo tornam-nas responsáveis perante a sociedade (ROSA et al., 2011).

Conforme Wang e Bernell (2013) a evidenciação ambiental corporativa, pode ser entendida como o conjunto de informações que se relacionam com as atividades de uma empresa referente á parte ambiental. Para tanto, as instituições internacionais desenvolveram padrões que buscam incentivar a adoção pelas empresas da divulgação ambiental. Um dos padrões internacionais mais populares é o Global Reporting Initiative (GRI), este que dispõe um conjunto de diretrizes para a divulgação de informações econômicas, ambientais e sociais.

Clarkson et al. (2013) salientam que estabelecer o conteúdo informativo das divulgações ambientais é fundamental para a responsabilidade social empresarial, pois a divulgação é resultado de equilíbrio de um processo de seleção. Lu e Abeysekera (2014) abordam que a divulgação social e ambiental é considerada um diálogo entre as empresas e seus stakeholders e o cumprimento da responsabilidade social da empresa para com estes. Deste modo, a evidenciação ambiental das empresas pode ser vista como uma estratégia de gestão eficaz para desenvolver e manter relações satisfatórias com as partes interessadas.

Em relação às divulgações ambientais, Iatridis (2013) destaca que devem incluir questões fundamentais do meio ambiente, impacto no desempenho da empresa, posição futura e políticas ambientais adotadas. No que diz respeito às divulgações ambientais adicionais, estas incluem as penalidades para o não cumprimento de determinada regulamentação, investimentos para a proteção do meio ambiente, cumprimento de padrões de qualidade internos e externos, projetos de sustentabilidade, dentre outros aspectos.

Diante do contexto supracitado, Rosa et al. (2010) salientam que as mudanças ocorridas no ecossistema global, interessa a comunidade científica, sociedade, governo, empresas e os ambientalistas, com o objetivo de solucionar e reduzir os reflexos do desequilíbrio ecológico. Em relação a esse problema, as empresas passaram a ser pressionadas a fornecer informações que passem a auxiliar as partes interessadas a avaliar o relacionamento da empresa para com o meio ambiente e sociedade. Assim, com o objetivo de aperfeiçoar a comunicação entre as empresas e sociedade é de que surgem os estudos referentes à evidenciação ambiental.

\section{PROCEDIMENTOS METODOLÓGICOS}

O presente estudo caracteriza-se como descritivo, documental e quantitativo. Descritivo por descrever a posição das empresas brasileiras listadas no IBrX-100 da BM\&FBovespa nos rankings formados pelo método da entropia e método TOPSIS. Documental visto que as informações ambientais foram coletadas nos RS e RA. A pesquisa é quantitativa, ao utilizar métodos estatísticos para o tratamento dos dados.

A população da presente pesquisa constitui-se de empresas pertencentes ao Índice Brasil 100 (IBrX-100) listadas na BM\&FBovespa, perfazendo um total de 100 companhias. No entanto, dentre essas empresas, a Bradesco, Klabin e Oi apresentam-se listadas duas vezes. Assim, 97 empresas compõem a amostra deste estudo.

Os RS e RA foram baixados diretamente do sítio das empresas analisadas e nestes relatórios verificou-se os seguintes aspectos ambientais: Emissões, Efluentes Líquidos, Resíduos, Produtos/Serviços e Transporte, estes que apresentam critérios e subcritérios ambientais, perfazendo um total de 33 itens que foram verificados nos relatórios. A definição destas variáveis partiu das diretrizes propostas pelo GRI (2013) e do estudo desenvolvido por Rosa et al. (2014). Para tanto, elaborou-se uma planilha eletrônica de excel, na qual foi preenchido o respectivo nível de cada critério e subcritério analisado de cada empresa.

Destaca-se que cada critério e subcritério ambiental apresentam diferentes escalas ordinais, estas que visam expressar ordem entre os níveis, de acordo com o desempenho de 
cada critério, permitindo a atribuição de até oito níveis. Dessa forma, utilizou-se o GRI (2013) e o estudo de Rosa et al. (2014) neste estudo, para interpretar quais as informações ambientais são evidenciadas pelas empresas analisadas em seus RS e RA, com o intuito da identificação do nível da informação ambiental para posteriormente elaborar os rankings. Os dados coletados são do período de 2010 a 2013.

Para a realização da análise dos dados utilizou-se do método da entropia, do qual se obtém um peso da informação, este que foi utilizado para a aplicação do método TOPSIS para formar os rankings, possibilitando a identificação das empresas que apresentam o melhor grau de evidenciação dos impactos ambientais. Para melhor entendimento de como funciona o modelo da Entropia e o modelo de análise multicritério TOPSIS, na seção a seguir apresentase mais detalhadamente cada método.

\subsection{Modelo da Entropia}

A entropia foi utilizada neste estudo, para calcular o peso que cada variável possui no conjunto de aspectos ambientais, permitindo a aplicação do método multicritério TOPSIS e, assim, classificar os cenários de forma adequada. O procedimento da entropia é explicado por Zeleny (1982), conforme segue:

Sejam, $\left.d_{i}=d_{i}^{1}, d_{i}^{2}, \ldots, d_{i}^{m}\right)$ os valores normalizados, em que o critério máximo $d_{i}^{k}=\frac{x_{i}^{k}}{x_{i}^{*}}$, caracteriza o conjunto $\mathrm{D}$, em termos do i-ésimo atributo. Deste modo, busca-se $D_{i}=\sum_{k=1}^{m} d_{i}^{k} ; i=1,2, \ldots, n$. Após esse passo, busca-se a medida de entropia do contraste de intensidade para o i-ésimo atributo, calculado por $e\left(d_{i}\right)=-\alpha \sum_{k=1}^{m} \frac{d_{i}^{k}}{D_{i}} \operatorname{Ln}\left(\frac{d_{i}^{k}}{D_{i}}\right)$, em que $\alpha=\frac{1}{e_{\max }}>0$ e $e_{\max }=\operatorname{Ln}(m)$. Assim, tem-se que $0 \leq d_{i}^{k} \leq 1$ e $d_{i}^{k} \geq 0$. Caso todos os $d_{i}^{k}$ forem iguais para um dado $\mathrm{i}$, então $\frac{d_{i}^{k}}{D_{i}}=\frac{1}{n} \mathrm{e} \mathrm{e}\left(\mathrm{d}_{\mathrm{i}}\right)$ assumem valor máximo, isto é, $e_{\max }=$ $\operatorname{Ln}(m)$. Ao se fixar $\alpha=\frac{1}{e_{\max }}$, determina-se $0 \leq e\left(d_{i}\right) \leq 1$ para todos os $\mathrm{d}_{\mathrm{i}}$ 's. Essa normalização é necessária para efeito comparativo. A entropia total de D é definida por $E=\sum_{i=1}^{n} e\left(d_{i}\right)$.

Observa-se que quanto maior for o e $\left(\mathrm{d}_{\mathrm{i}}\right)$, menor será a informação transmitida pelo iésimo atributo. Caso $e\left(d_{i}\right)=e_{\max }=\operatorname{Ln}(m)$, então o i-ésimo atributo não transmite informações e pode ser removido da análise decisória. Assim o peso $\tilde{\lambda}_{\mathrm{i}}$ ser inversamente relacionado a e $\left(\mathrm{d}_{\mathrm{i}}\right)$, usa-se 1-e $\left(\mathrm{d}_{\mathrm{i}}\right)$ ao invés de e $\left(\mathrm{d}_{\mathrm{i}}\right)$ e normaliza-se para assegurar que $0 \leq \tilde{\lambda}_{\mathrm{i}} \leq$ 1e $\sum_{i=1}^{n} \tilde{\lambda}_{\mathrm{i}}=1$. Para tanto, a entropia da informação pode ser representada pela seguinte expressão: $\tilde{\lambda}_{\mathrm{i}}=\frac{1}{n-E}\left[1-e\left(d_{i}\right)\right]=\frac{\left[1-e\left(d_{i}\right)\right]}{n-E}$. Apresentado o modelo da Entropia, na seção a seguir apresentam-se os passos do modelo de análise multicritério TOPSIS.

\subsection{Technique for Order Preference by Similarity to an Ideal Solution - TOPSIS}

A Technique for Order Preference by Similarity to an Ideal Solution (TOPSIS), isto é, modelo de análise multicritério TOPSIS, foi proposto por Hwang e Yoon, no ano de 1981 (ERTUGRUL; KARAKASOGLU, 2009). A ideia inicial partiu do conceito de solução de compromisso, para escolher a melhor alternativa e mais próxima para a solução ideal positiva (solução ótima) e a mais distante da solução ideal negativa (solução inferior). Na sequência determina-se o melhor da classificação, obtendo assim, a melhor alternativa (TZENG; HUANG, 2011).

De acordo com Wu, Tzeng e Chen (2009) e Tzeng e Huang (2011), o modelo TOPSIS baseia-se no conceito de que as melhores alternativas e escolhas devem ser a distância considerada mais curta entre a solução ideal positiva e a mais distante à solução ideal 
negativa. Para tanto, conforme Bulgurcu (2012) este método considera a distância entre ambos os lados para a classificação dos elementos analisados.

Segundo Ertugrul e Karakasoglu (2009) a solução ideal pode ser positiva e negativa. A solução ideal positiva passa a maximizar o critério de benefícios e minimiza os critérios relacionados ao custo. Já na solução ideal negativa, ocorre o contrário, maximiza os critérios de custo e minimiza os critérios voltados aos benefícios. Deste modo, a solução ideal positiva é aquela que apresenta todos os melhores valores possíveis e a solução ideal negativa é constituída por todos os valores piores que podem ser atingidos.

Nesse sentido, Wu, Tzeng e Chen (2009), ressaltam que a melhor alternativa não somente deve ser a distância considerada mais curta a partir da solução ideal positiva, mas também deve-se levar em conta a maior distância a partir da solução ideal negativa.

Os procedimentos do método TOPSIS iniciam com a realização de uma matriz de dados original, que utiliza critérios de valor para cada alternativa. Dessa forma, o TOPSIS transforma essa matriz original em uma matriz considerada normalizada e apresenta as etapas descritas na sequência (BULGURCU, 2012).

O modelo TOPSIS inicia com uma matriz de decisão constituída por alternativas e critérios:

$$
\mathrm{A}=\left[\begin{array}{ccc}
\mathrm{v}_{11} & \cdots & \mathrm{v}_{1 \mathrm{n}} \\
\vdots & \ddots & \vdots \\
\mathrm{v}_{\mathrm{m} 1} & \cdots & \mathrm{v}_{\mathrm{mn}}
\end{array}\right]
$$

Esta técnica apresenta três passos. O primeiro diz respeito ao cálculo das soluções ideais positivas $\mathrm{A}^{+}$(benefícios) e das soluções ideais negativas $\mathrm{A}^{-}$(custos), da seguinte forma: $A^{+}=\left(p_{1}^{+}, p_{2}^{+}, \ldots, p_{m}^{+}\right)$e $A^{-}=\left(p_{1}^{-}, p_{2}^{-}, \ldots, p_{m}^{-}\right)$,

Onde:

$$
\mathrm{p}_{\mathrm{j}}^{+}=\left\{\operatorname{Max}_{\mathrm{i}} \mathrm{P}_{\mathrm{ij}}, \mathrm{j} \in \mathrm{J}_{1} ; \operatorname{Min}_{\mathrm{i}} \mathrm{P}_{\mathrm{ij}}, \mathrm{j} \in \mathrm{J}_{2}\right\}
$$

$\mathrm{p}_{\mathrm{j}}^{-}=\left\{\operatorname{Min}_{\mathrm{i}} \mathrm{p}_{\mathrm{ij}}, \mathrm{j} \in \mathrm{J}_{1} ; \operatorname{Max}_{\mathrm{i}} \mathrm{p}_{\mathrm{ij}}, \mathrm{j} \in \mathrm{J}_{2}\right\}$,

Onde: $\mathrm{J}_{1}$ e $\mathrm{J}_{2}$ representam respectivamente o critério de benefício e custo.

O segundo passo consiste no cálculo das distâncias euclidianas, isto é, cálculo da medida de separação. Este cálculo das distâncias euclidianas entre os benefícios é então dado por:

$d^{+}=\sqrt{\sum_{j=1}^{n} w_{j}\left(p_{j}^{+}-p_{i j}\right)^{2}}, \operatorname{com~i}=1, \ldots, \mathrm{m} \mathrm{e} d^{-}=\sqrt{\sum_{j=1}^{n} w_{j}\left(p_{j}^{-}-p_{i j}\right)^{2}}, \operatorname{com} \mathrm{i}=1, \ldots, \mathrm{m}$.

Nesta pesquisa, os pesos foram utilizados com valores de $w_{i}=1$. Já o terceiro passo do método TOPSIS é o cálculo da proximidade relativa em relação à solução ideal, conforme segue:

$$
\xi_{\mathrm{i}}=\frac{\mathrm{d}_{\mathrm{i}}^{-}}{\mathrm{d}_{\mathrm{i}}^{+}+\mathrm{d}_{\mathrm{i}}^{-}}
$$

Por fim, após a realização destes passos do TOPSIS o ranking é elaborado de modo que a empresa mais próxima da solução ideal é designada como a primeira colocada no ranking e assim sucessivamente. Bulgurcu (2012) salienta ainda, que o ranking da ordem de preferência é realizado de acordo com a ordem decrescente da solução ideal. Os resultados 
dos modelos aplicados para as empresas brasileiras listadas no IBrX-100 da BM\&FBovespa são apresentados na seção descrição e análise dos dados.

\section{DESCRIÇÃO E ANÁLISE DOS DADOS}

Apresenta-se nesta seção a análise dos rankings das empresas analisadas a partir dos aspectos ambientais: Emissões, Efluentes, Resíduos, Produtos/Serviços e Transporte e, por fim apresenta-se um ranking geral, este que engloba os cinco aspectos ambientais analisados. Os rankings foram gerados por meio da aplicação da entropia e após o método TOPSIS. Na Tabela 1 apresenta-se o ranking de evidenciação dos impactos ambientais sobre o aspecto Emissões, contendo apenas as empresas que obtiveram as dez primeiras colocações no ranking final. O ranking final foi elaborado a partir de um sistema de pontos corridos.

Tabela 1 - Ranking de evidenciação dos impactos ambientais sobre o aspecto Emissões

\begin{tabular}{|c|c|c|c|c|c|c|c|c|c|}
\hline \multirow{2}{*}{ Empresas } & \multicolumn{2}{|c|}{2010} & \multicolumn{2}{|c|}{2011} & \multicolumn{2}{|c|}{2012} & \multicolumn{2}{|c|}{2013} & \multirow{2}{*}{$\begin{array}{c}\text { Ranking } \\
\text { Final }\end{array}$} \\
\hline & Índice & Posição & Índice & Posição & Índice & Posição & Índice & Posição & \\
\hline P. Açúcar CBD & 0,5697 & $5^{a}$ & 0,8044 & $1^{\mathrm{a}}$ & 0,7322 & $3^{a}$ & 0,7569 & $2^{a}$ & $1^{\mathrm{a}}$ \\
\hline Ecorodovias & 0,8487 & $1^{\mathrm{a}}$ & 0,5962 & $5^{a}$ & 0,7482 & $2^{a}$ & 0,5711 & $7^{a}$ & $2^{a}$ \\
\hline Energias Br. & 0,6266 & $3^{a}$ & 0,6267 & $4^{a}$ & 0,7297 & $4^{a}$ & 0,6132 & $5^{a}$ & $3^{a}$ \\
\hline Duratex & 0,7168 & $2^{a}$ & 0,6576 & $3^{a}$ & 0,6309 & $8^{a}$ & 0,6172 & $4^{a}$ & $4^{a}$ \\
\hline Braskem & 0,4216 & $12^{\mathrm{a}}$ & 0,3804 & $15^{\mathrm{a}}$ & 0,6162 & $9^{a}$ & 0,6787 & $3^{a}$ & $5^{\mathrm{a}}$ \\
\hline Copel & 0,6056 & $4^{a}$ & 0,7666 & $2^{a}$ & 0,6019 & $10^{\mathrm{a}}$ & 0,3263 & $24^{a}$ & $6^{a}$ \\
\hline Natura & 0,4572 & $9^{a}$ & 0,4957 & $9^{a}$ & 0,6886 & $5^{a}$ & 0,3589 & $19^{\mathrm{a}}$ & $7^{a}$ \\
\hline Even & 0,354 & $17^{\mathrm{a}}$ & 0,5518 & $7^{a}$ & 0,5367 & $13^{a}$ & 0,5065 & $11^{\mathrm{a}}$ & $8^{a}$ \\
\hline BM\&FBovespa & 0,3416 & $18^{\mathrm{a}}$ & 0,3352 & $21^{a}$ & 0,7704 & $1^{\mathrm{a}}$ & 0,5533 & $9^{a}$ & $9^{a}$ \\
\hline Petrobrás & 0,5041 & $8^{a}$ & 0,4733 & $11^{\mathrm{a}}$ & 0,3885 & $22^{a}$ & 0,3969 & $14^{a}$ & $10^{\mathrm{a}}$ \\
\hline
\end{tabular}

Fonte: Dados da pesquisa.

De acordo com os dados apresentados na Tabela 1 referente ao ranking de evidenciação dos impactos ambientais sobre emissões nota-se que as três empresas mais bem colocadas no ranking foram às empresas P. Açúcar CBD, Ecorodovias e Energias Br. A empresa P. Açúcar CBD no ano de 2010 apresentou uma evidenciação ambiental em seu RS e RA que a permitiu constar na quinta posição no ranking. Em 2011 passou a liderar o ranking, porém no ano de 2012 e 2013 decaiu para a terceira e segunda colocação respectivamente. Esta empresa do ramo alimentício tem se destacado nos quatro anos analisados, visto que as emissões de gases relacionadas às operações da empresa que provocam impacto do aquecimento global são mitigadas por meio do mapeamento das emissões, a partir de inventário de carbono.

A empresa Ecorodovias no ano de 2010 apresentava-se como a primeira colocada no ranking, em 2011 passou a ocupar a quinta posição, no ano de 2012 subiu de posição novamente, mas para a segunda colocação. No ano de 2013 evidenciou menos informações passando dessa forma a ocupar a sétima posição. A empresa Energias Br., terceira colocada no ranking final, manteve um grau de divulgação dos impactos ambientais sobre emissões praticamente no mesmo nível no período de 2010 a 2013. Na tabela 2, apresenta-se o ranking de evidenciação dos impactos ambientais sobre o aspecto Efluentes.

Tabela 2 - Ranking de evidenciação dos impactos ambientais sobre o aspecto Efluentes

\begin{tabular}{l|c|c|c|c|c|c|c|c|c|c}
\hline \multirow{2}{*}{ Empresas } & \multicolumn{2}{c|}{2010} & \multicolumn{2}{c|}{2011} & \multicolumn{2}{c|}{2012} & \multicolumn{2}{c|}{2013} & Ranking \\
\cline { 2 - 11 } & Índice & Posição & Índice & Posição & Índice & Posição & Índice & Posição & Final \\
\hline Cemig & 0,3421 & $8^{\mathrm{a}}$ & 0,4366 & $3^{\mathrm{a}}$ & 0,6175 & $6^{\mathrm{a}}$ & 0,5976 & $4^{\mathrm{a}}$ & $1^{\mathrm{a}}$ \\
\hline BRF - Brasil Foods & 1 & $1^{\mathrm{a}}$ & 0,5861 & $1^{\mathrm{a}}$ & 1 & $1^{\mathrm{a}}$ & 0 & $29^{\mathrm{a}}$ & $2^{\mathrm{a}}$ \\
\hline Fibria & 0,3858 & $7^{\mathrm{a}}$ & 0,4041 & $8^{\mathrm{a}}$ & 0,4585 & $11^{\mathrm{a}}$ & 0,3437 & $7^{\mathrm{a}}$ & $3^{\mathrm{a}}$ \\
\hline Duratex & 0,5491 & $4^{\mathrm{a}}$ & 0,5736 & $2^{\mathrm{a}}$ & 0,6193 & $4^{\mathrm{a}}$ & 0,1106 & $24^{\mathrm{a}}$ & $4^{\mathrm{a}}$
\end{tabular}




\begin{tabular}{l|c|c|c|c|c|c|c|c|c} 
Petrobrás & 0,4126 & $6^{\mathrm{a}}$ & 0,4308 & $4^{\mathrm{a}}$ & 0,3868 & $17^{\mathrm{a}}$ & 0,3227 & $12^{\mathrm{a}}$ & $5^{\mathrm{a}}$ \\
\hline Eletrobrás & 0 & $30^{\mathrm{a}}$ & 0,3569 & $13^{\mathrm{a}}$ & 0,6175 & $7^{\mathrm{a}}$ & 0,9393 & $1^{\mathrm{a}}$ & $6^{\mathrm{a}}$ \\
\hline Klabin & 0,0803 & $22^{\mathrm{a}}$ & 0,4129 & $6^{\mathrm{a}}$ & 0,5042 & $8^{\mathrm{a}}$ & 0,3217 & $16^{\mathrm{a}}$ & $7^{\mathrm{a}}$ \\
\hline Jbs & 0,0756 & $27^{\mathrm{a}}$ & 0,1543 & $24^{\mathrm{a}}$ & 0,7044 & $2^{\mathrm{a}}$ & 0,7139 & $2^{\mathrm{a}}$ & $8^{\mathrm{a}}$ \\
\hline Natura & 0,1504 & $17^{\mathrm{a}}$ & 0,4152 & $5^{\mathrm{a}}$ & 0,4510 & $12^{\mathrm{a}}$ & 0,1316 & $23^{\mathrm{a}}$ & $9^{\mathrm{a}}$ \\
\hline Weg & 0 & $30^{\mathrm{a}}$ & 0,3583 & $12^{\mathrm{a}}$ & 0,4006 & $14^{\mathrm{a}}$ & 0,3753 & $6^{\mathrm{a}}$ & $10^{\mathrm{a}}$ \\
\hline
\end{tabular}

Fonte: Dados da pesquisa.

Segundo a Tabela 2, as empresas mais bem colocadas no ranking de evidenciação dos impactos ambientais sobre efluentes foram às empresas Cemig, BRF - Brasil Foods, e Fibria. Para tanto, A empresa Cemig no ano de 2010 constava na oitava posição no ranking, em 2011 passou para a terceira posição, já em 2012 divulgou menos informações ambientais sobre efluentes passando para a sexta colocação e em 2013 novamente subiu de posição, ocupando o quarto lugar. Já no ranking final liderou.

A empresa BRF - Brasil Foods, nos anos de 2010, 2011 e 2012 foi à primeira colocada no ranking, porém em 2013 decaiu para a 29a posição, o que indica que no ano de 2013 não divulgou RS. No ranking final ficou na segunda colocação. Este resultado evidencia que esta empresa do ramo alimentício pode ter esta preocupação com o lançamento de efluentes, devido ao fato de seu maior impacto ambiental estar diretamente relacionado com a utilização, bem como, consumo da água.

Destaca-se ainda a empresa Fibria, pois no ano de 2010 constava na sétima colocação, em 2011 subiu para a $8^{\mathrm{a}}$ posição, no ano de 2012 ficou na $11^{\mathrm{a}}$ posição e novamente apresentou-se na sétima colocação no ano de 2013. No que tange o ranking final, ficou na terceira posição referente à evidenciação dos impactos ambientais sobre efluentes. Por meio da Tabela 3 apresentam-se os rankings de evidenciação dos impactos ambientais sobre o aspecto Resíduos.

Tabela 3 - Ranking de evidenciação dos impactos ambientais sobre o aspecto Resíduos

\begin{tabular}{|c|c|c|c|c|c|c|c|c|c|}
\hline \multirow{2}{*}{ Empresas } & \multicolumn{2}{|c|}{2010} & \multicolumn{2}{|c|}{2011} & \multicolumn{2}{|c|}{2012} & \multicolumn{2}{|c|}{2013} & \multirow{2}{*}{$\begin{array}{c}\text { Ranking } \\
\text { Final }\end{array}$} \\
\hline & Índice & Posição & Índice & Posição & Índice & Posição & Índice & Posição & \\
\hline Ecorodovias & 0,7346 & $4^{\mathrm{a}}$ & 0,5128 & $9^{a}$ & 0,7814 & $1^{\mathrm{a}}$ & 0,7834 & $3^{\mathrm{a}}$ & 1 \\
\hline Energias Br. & 0,7604 & $2^{a}$ & 0,7761 & $1^{\mathrm{a}}$ & 0,7572 & $5^{a}$ & 0,7592 & $10^{\mathrm{a}}$ & 2 \\
\hline BM\&FBovespa & 0,6683 & $6^{\mathrm{a}}$ & 0,5514 & $6^{\mathrm{a}}$ & 0,7572 & $2^{a}$ & 0,7592 & $7^{\mathrm{a}}$ & 3 \\
\hline Duratex & 0,7346 & $3^{\mathrm{a}}$ & 0,7649 & $2^{a}$ & 0,5391 & $11^{\mathrm{a}}$ & 0,7802 & $5^{a}$ & 3 \\
\hline P. Açúcar CBD & 0,4166 & $8^{a}$ & 0,4915 & $11^{\mathrm{a}}$ & 0,4781 & $17^{\mathrm{a}}$ & 0,7834 & $4^{\mathrm{a}}$ & 5 \\
\hline Cemig & 0,3620 & $12^{\mathrm{a}}$ & 0,3582 & $20^{a}$ & 0,7572 & $3^{a}$ & 0,7592 & $8^{a}$ & 6 \\
\hline Braskem & 0,3546 & $14^{\mathrm{a}}$ & 0,3289 & $23^{a}$ & 0,6579 & $7^{a}$ & 0,7592 & $6^{a}$ & 7 \\
\hline Copel & 0,1535 & $26^{\mathrm{a}}$ & 0,6403 & $4^{a}$ & 0,5790 & $10^{\mathrm{a}}$ & 0,5225 & $11^{\mathrm{a}}$ & 8 \\
\hline Petrobrás & 0,6841 & $5^{a}$ & 0,7264 & $3^{a}$ & 0,4128 & $24^{a}$ & 0,4143 & $21^{a}$ & 9 \\
\hline Even & 0,2364 & $18^{\mathrm{a}}$ & 0,4915 & $10^{a}$ & 0,4781 & $15^{\mathrm{a}}$ & 0,4731 & $12^{a}$ & 10 \\
\hline
\end{tabular}

Fonte: Dados da pesquisa.

Conforme a Tabela 3, as empresas que se encontram mais bem colocadas no ranking de evidenciação dos impactos ambientais sobre resíduos foram as empresas Ecorodovias, Energias Br. e BM\&FBovespa. A empresa Ecorodovias, no ano de 2010 estava na quarta colocação, em 2011 passou para a 9a , em 2012 liderou o ranking e ainda, em 2013 apresentava-se na terceira posição.

Nesse sentido, a empresa Energias Br., no ano de 2010 constava na segunda posição do ranking, em 2011 liderou, já em 2012 e 2013 passou para a quinta e décima posição respectivamente. A empresa BM\&FBovespa nos anos de 2010 e 2011 manteve-se na sexta colocação no ranking, em 2012 passou para a segunda posição e no ano de 2013 decaiu para a sétima colocação no ranking de evidenciação dos impactos ambientais sobre resíduos. A 
Tabela 4 apresenta os rankings de evidenciação dos impactos ambientais sobre o aspecto Produtos e Serviços.

Tabela 4 - Ranking de evidenciação dos impactos ambientais sobre o aspecto Produtos e Serviços

\begin{tabular}{|c|c|c|c|c|c|c|c|c|c|}
\hline \multirow{2}{*}{ Empresas } & \multicolumn{2}{|c|}{2010} & \multicolumn{2}{|c|}{2011} & \multicolumn{2}{|c|}{2012} & \multicolumn{2}{|c|}{2013} & \multirow{2}{*}{$\begin{array}{c}\text { Ranking } \\
\text { Final }\end{array}$} \\
\hline & Índice & Posição & Índice & Posição & Índice & Posição & Índice & Posição & \\
\hline Ecorodovias & 0,6038 & $6^{a}$ & 0,7566 & $3^{a}$ & 0,7223 & $6^{a}$ & 0,9935 & $2^{a}$ & $1^{\mathrm{a}}$ \\
\hline Duratex & 0,6532 & $4^{\mathrm{a}}$ & 0,7566 & $2^{\mathrm{a}}$ & 0,6002 & $10^{\mathrm{a}}$ & 0,8963 & $3^{\mathrm{a}}$ & $2^{a}$ \\
\hline Copel & 0,6521 & $5^{a}$ & 0,8227 & $1^{\mathrm{a}}$ & 0,4730 & $18^{\mathrm{a}}$ & 0,7192 & $6^{a}$ & $3^{\mathrm{a}}$ \\
\hline P. Açúcar CBD & 0,6595 & $3^{\mathrm{a}}$ & 0,6318 & $7^{\mathrm{a}}$ & 0,5658 & $11^{\mathrm{a}}$ & 0,6476 & $10^{\mathrm{a}}$ & $4^{\mathrm{a}}$ \\
\hline Energias Br. & 0,7104 & $2^{a}$ & 0,7547 & $5^{a}$ & 0,5199 & $14^{\mathrm{a}}$ & 0,5883 & $14^{\mathrm{a}}$ & $5^{a}$ \\
\hline Natura & 0,4598 & $10^{\mathrm{a}}$ & 0,6792 & $6^{a}$ & 0,7439 & $4^{a}$ & 0,5883 & $15^{\mathrm{a}}$ & $5^{a}$ \\
\hline Copasa & 0,5996 & $7^{a}$ & 0,7547 & $4^{a}$ & 0,6538 & $8^{a}$ & 0,1229 & $42^{a}$ & $7^{a}$ \\
\hline Aes Tiete & 0,5136 & $8^{a}$ & 0,5425 & $11^{\mathrm{a}}$ & 0,4409 & $23^{a}$ & 0,4654 & $20^{\mathrm{a}}$ & $8^{a}$ \\
\hline Banco Bradesco & 0,1386 & $25^{\mathrm{a}}$ & 0,5879 & $10^{\mathrm{a}}$ & 0,5175 & $15^{\mathrm{a}}$ & 0,5883 & $13^{a}$ & $9^{a}$ \\
\hline Eletrobrás & 0,0748 & $32^{a}$ & 0,4096 & $20^{a}$ & 0,7223 & $7^{a}$ & 0,8963 & $4^{a}$ & $9^{a}$ \\
\hline
\end{tabular}

Fonte: Dados da pesquisa.

Os resultados expostos na Tabela 4 indicam que as empresas mais bem colocadas no ranking de evidenciação dos impactos ambientais sobre produtos e serviços (com base na posição média do período) foram às empresas Ecorodovias, Duratex e Copel, visto que estas empresas tiveram um grau de evidenciação ambiental, em média, entre $66 \%$ e $76 \%$, isto é, apresentaram o maior grau de evidenciação ambiental durante o período analisado.

A empresa Ecorodovias no ano de 2010 constava na sexta posição e em 2011 subiu para a terceira. Já em 2012 voltou a ocupar a sexta posição e no ano de 2013 passou para a segunda posição no ranking. A empresa Duratex no ano de 2010 ocupava a quarta posição no ranking, em 2011 passou para a segunda, já em 2012 decaiu para a décima posição, o que demonstra a diminuição de divulgação ambiental sobre os produtos e serviços, porém em 2013 subiu para a terceira colocação. Contudo, a empresa Copel de quinta colocada em 2010 passou a liderar o ranking em 2011, entretanto em 2012 decaiu para a posição $18^{a}$ e no ano de 2013 reagiu a sua divulgação ambiental, passando deste modo para a sexta posição. A Tabela 5 apresenta os rankings de evidenciação dos impactos ambientais sobre o aspecto Transporte.

Tabela 5 - Ranking de evidenciação dos impactos ambientais sobre o aspecto Transporte

\begin{tabular}{|c|c|c|c|c|c|c|c|c|c|}
\hline \multirow{2}{*}{ Empresas } & \multicolumn{2}{|c|}{2010} & \multicolumn{2}{|c|}{2011} & \multicolumn{2}{|c|}{2012} & \multicolumn{2}{|c|}{2013} & \multirow{2}{*}{$\begin{array}{c}\text { Ranking } \\
\text { Final }\end{array}$} \\
\hline & Índice & Posição & Índice & Posição & Índice & Posição & Índice & Posição & \\
\hline Copel & 0,8417 & $1^{\mathrm{a}}$ & 0,9108 & $1^{\mathrm{a}}$ & 0,7251 & $3^{a}$ & 0,1981 & $15^{\mathrm{a}}$ & $1^{\mathrm{a}}$ \\
\hline Natura & 0,0981 & $16^{\mathrm{a}}$ & 0,7889 & $2^{a}$ & 0,5269 & $4^{a}$ & 0,2031 & $10^{\mathrm{a}}$ & $2^{a}$ \\
\hline Petrobrás & 0,1095 & $7^{a}$ & 0,1085 & $11^{\mathrm{a}}$ & 0,1034 & $18^{\mathrm{a}}$ & 0,6918 & $3^{a}$ & $3^{a}$ \\
\hline Itauunibanco & 0 & $18^{\mathrm{a}}$ & 0,1064 & $12^{\mathrm{a}}$ & 0,4482 & $5^{a}$ & 0,4956 & $5^{a}$ & $4^{a}$ \\
\hline P. Açúcar CBD & 0,1957 & $6^{\mathrm{a}}$ & 0,1988 & $9^{a}$ & 0,0928 & $25^{a}$ & 0,9195 & $1^{\mathrm{a}}$ & $5^{a}$ \\
\hline Ecorodovias & 0 & $18^{\mathrm{a}}$ & 0,2114 & $7^{a}$ & 0,2013 & $15^{a}$ & 0,2035 & $8^{a}$ & $6^{a}$ \\
\hline Banco Brasil & 0 & $18^{\mathrm{a}}$ & 0 & $21^{a}$ & 0,3641 & $12^{\mathrm{a}}$ & 0,5000 & $4^{a}$ & $7^{\mathrm{a}}$ \\
\hline Copasa & 0 & $18^{\mathrm{a}}$ & 0 & $21^{a}$ & 0,7251 & $2^{a}$ & 0,1981 & $14^{\mathrm{a}}$ & $7^{a}$ \\
\hline Eletropaulo & 0,4871 & $3^{a}$ & 0,4966 & $6^{\mathrm{a}}$ & 0,0976 & $20^{a}$ & 0,0991 & $26^{a}$ & $7^{a}$ \\
\hline Lojas Renner & 0 & $18^{\mathrm{a}}$ & 0 & $21^{a}$ & 0,4444 & $11^{\mathrm{a}}$ & 0,4937 & $6^{a}$ & $10^{\mathrm{a}}$ \\
\hline
\end{tabular}

Fonte: Dados da pesquisa.

De acordo com os dados expostos na Tabela 5, as empresas mais bem colocadas no ranking de evidenciação dos impactos ambientais sobre transporte foram às empresas Copel, Natura e Petrobrás. Para tanto, a empresa Copel liderava o ranking nos anos de 2010 e 2011. Já em 2012 decaiu para a terceira posição e em 2013 passou a divulgar menos informações sobre os impactos dos transportes passando para a $15^{\mathrm{a}}$ colocação. Este resultado evidencia que 
a frota de veículos destinada às atividades operacionais e ao transporte de pessoas a serviço da entidade é adquirida com prioridade para os modelos que atendam às exigências legais quanto à emissão de poluentes no meio ambiente.

A empresa Natura no ano de 2010 apresentava menos informações sobre o transporte nos seus relatórios, visto que constava na $16^{a}$ posição no ranking, já em 2011 passou para a segunda colocada, no ano de 2012 a quarta e em 2013 decaiu para a décima posição. Esta empresa, a partir de 2013, foi a primeira a ter em sua frota um ônibus executivo à base de etanol, o que ocorria apenas no transporte público. Este ônibus apresenta uma emissão de $88 \%$ menor, quando comparado ao veículo tradicional. Já a empresa Petrobrás no ano de 2010 era a sétima colocada no ranking, em 2011 a $11^{\text {a }}$ colocada, em 2012 passou para a $18^{\mathrm{a}}$ posição e em 2013 ocupou a terceira colocação no ranking de evidenciação dos impactos ambientais sobre transporte. Na Tabela 6 apresenta-se o ranking geral de evidenciação dos impactos ambientais analisados.

Tabela 6 - Ranking geral de evidenciação dos impactos ambientais

\begin{tabular}{l|c|c|c|c|c|c|c|c|c|c}
\multirow{2}{*}{ Empresas } & \multicolumn{2}{c|}{$\mathbf{2 0 1 0}$} & \multicolumn{2}{c|}{$\mathbf{2 0 1 1}$} & \multicolumn{2}{c|}{$\mathbf{2 0 1 2}$} & \multicolumn{3}{c|}{$\mathbf{2 0 1 3}$} & Ranking \\
\cline { 2 - 11 } & Índice & Posição & Índice & Posição & Índice & Posição & Índice & Posição & Final \\
\hline Ecorodovias & 0,6264 & $3^{\mathrm{a}}$ & 0,5346 & $5^{\mathrm{a}}$ & 0,6393 & $1^{\mathrm{a}}$ & 0,5735 & $6^{\mathrm{a}}$ & $1^{\mathrm{a}}$ \\
\hline Duratex & 0,6506 & $2^{\mathrm{a}}$ & 0,6385 & $2^{\mathrm{a}}$ & 0,5656 & $8^{\mathrm{a}}$ & 0,5595 & $8^{\mathrm{a}}$ & $2^{\mathrm{a}}$ \\
\hline Energias Br. & 0,5825 & $4^{\mathrm{a}}$ & 0,6026 & $3^{\mathrm{a}}$ & 0,5830 & $6^{\mathrm{a}}$ & 0,5465 & $9^{\mathrm{a}}$ & $3^{\mathrm{a}}$ \\
\hline P. Açúcar CBD & 0,4621 & $7^{\mathrm{a}}$ & 0,5521 & $4^{\mathrm{a}}$ & 0,5132 & $11^{\mathrm{a}}$ & 0,6067 & $4^{\mathrm{a}}$ & $4^{\mathrm{a}}$ \\
\hline Copel & 0,5204 & $5^{\mathrm{a}}$ & 0,6769 & $1^{\mathrm{a}}$ & 0,5517 & $10^{\mathrm{a}}$ & 0,3928 & $12^{\mathrm{a}}$ & $5^{\mathrm{a}}$ \\
\hline BM\&FBovespa & 0,4288 & $9^{\mathrm{a}}$ & 0,3885 & $13^{\mathrm{a}}$ & 0,5532 & $9^{\mathrm{a}}$ & 0,4993 & $10^{\mathrm{a}}$ & $6^{\mathrm{a}}$ \\
\hline Natura & 0,3037 & $14^{\mathrm{a}}$ & 0,4788 & $9^{\mathrm{a}}$ & 0,6293 & $2^{\mathrm{a}}$ & 0,3526 & $17^{\mathrm{a}}$ & $7^{\mathrm{a}}$ \\
\hline Braskem & 0,2991 & $15^{\mathrm{a}}$ & 0,2653 & $23^{\mathrm{a}}$ & 0,6084 & $4^{\mathrm{a}}$ & 0,6604 & $2^{\mathrm{a}}$ & $8^{\mathrm{a}}$ \\
\hline Cemig & 0,2526 & $21^{\mathrm{a}}$ & 0,3173 & $17^{\mathrm{a}}$ & 0,6222 & $3^{\mathrm{a}}$ & 0,6223 & $3^{\mathrm{a}}$ & $8^{\mathrm{a}}$ \\
\hline Petrobrás & 0,4649 & $6^{\mathrm{a}}$ & 0,4876 & $7^{\mathrm{a}}$ & 0,3817 & $21^{\mathrm{a}}$ & 0,3650 & $14^{\mathrm{a}}$ & $10^{\mathrm{a}}$ \\
\hline
\end{tabular}

Fonte: Dados da pesquisa.

Conforme a Tabela 6, as empresas mais bem colocadas no ranking geral de evidenciação dos impactos ambientais analisados, isto é, emissões, efluentes, resíduos, produtos/serviços e transporte foram às empresas Ecorodovias, Duratex e Energias Br. Este resultado indica que estas empresas apresentaram a maior quantidade de informações sobre os critérios e subcritérios ambientais analisados em seus relatórios no período de 2010 a 2013.

A empresa Ecorodovias no período de 2010 encontrava-se como a terceira colocada no ranking, em 2011 a quinta posição. Já em 2012 foi à empresa que mais prestou informações ambientais em seus relatórios, pois passou a liderar o ranking, porém em 2013, decaiu para a sexta posição, o que indica que sua divulgação ambiental consequentemente diminui.

Deste modo, pode-se observar que a empresa Duratex no ano de 2010 e 2011 era à segunda colocada no ranking e nos anos de 2012 e 2013 sua evidenciação ambiental diminuiu, passando para a oitava posição. No que tange a terceira melhor empresa posicionada no ranking, têm-se a empresa Energias Br., esta que no ano de 2010 era a quarta colocada no ranking, em 2011 melhorou sua divulgação ambiental passando para a terceira posição. Contudo, nos anos de 2012 e 2013 novamente passou a divulgar menos informações ambientais, pois se apresentou como $6^{\mathrm{a}}$ e $9^{\mathrm{a}}$ colocada respectivamente.

Apesar da comunidade científica estar apresentando por meio de pesquisas a importância de diversos aspectos ambientais que devem ser divulgados pelas empresas, constata-se que os níveis de evidenciação ambiental ainda podem ser considerados baixos.

Os achados verificados nesta pesquisa corroboram com o exposto pela literatura, visto que no Brasil há presença significativa de empresas que apresentam a baixa adoção dos RS e RA, o que indica que o grau de evidenciação ambiental destas empresas é considerado baixo, 
assim como as empresas revelam poucas informações a seus interessados no que diz respeito à questão ambiental. Resultados semelhantes foram encontrados por Clarkson et al. (2008) e Lynch (2010), pois os autores consideram decepcionante o baixo nível de informação ambiental referente às orientações do GRI nos relatórios.

Bewley e Ly (2000) identificaram a partir de seus estudos que as empresas com maiores divulgações ambientais possuem maior propensão à poluição ambiental, resultado este que se assemelha a esta pesquisa. Zeng et al. (2012) aprimora esta questão salientando que as empresas que são consideradas potencialmente poluidoras e que já estão envolvidas com índices de divulgação ambiental, apresentam maiores níveis de evidenciação ambiental.

Por fim, Al-Tuwaijri, Christensen e Hughes II (2004) ressaltam que os gestores devem mudar a sua visão estratégica em relação ao desempenho ambiental, com vistas à melhoria da evidenciação ambiental, pois a divulgação das informações ambientais está relacionada com a qualidade da gestão.

\section{CONSIDERAÇÕES FINAIS}

O objetivo deste estudo de verificar o ranking de evidenciação dos impactos ambientais das empresas brasileiras listadas no IBrX-100 da BM\&FBovespa, a partir da utilização do modelo da entropia e método TOPSIS, foi alcançado, visto que foi possível analisar as posições que as empresas ocuparam nos rankings no período de 2010 a 2013.

Os resultados em relação aos rankings de evidenciação dos impactos ambientais revelaram que as empresas que se destacaram foram a Ecorodovias, Energias Br., Copel e Duratex, visto que apresentaram-se entre as três primeiras colocadas em mais de um dos rankings analisados, levando em conta o ranking final.

Para tanto, a empresa Ecorodovias ficou na segunda posição no ranking sobre o aspecto emissões e liderou o ranking sobre os aspectos resíduos, produtos/serviços e no ranking geral. A empresa Energias Br., no ranking referente o aspecto emissões constava na terceira colocação e no ranking sobre resíduos e geral apresentou-se na segunda e terceira posição respectivamente. No que tange a empresa Copel, no ranking relacionado aos produtos/serviços constava na terceira posição e liderou o ranking sobre o transporte. Por fim, a empresa Duratex ocupou a segunda colocação no ranking sobre o aspecto ambiental produtos/serviços e ranking geral de evidenciação dos impactos ambientais.

Verificou-se que as empresas que obtiveram as primeiras colocações no ranking geral, ocuparam posições intermediárias nos rankings sobre as emissões, efluentes, resíduos, produtos/serviços e transporte. Conclui-se, que uma maior evidenciação dos impactos ambientais sobre o aspecto emissões não indica, necessariamente, que a empresa possui maior evidenciação referente os resíduos e os demais aspectos analisados. Deste modo, é importante divulgar informações ambientais, visto que os RS e RA possibilitam que a empresa aprofunde o nível de evidenciação ambiental, e como consequência esta obterá diversos benefícios, como também a sociedade e o meio ambiente.

Sugere-se para pesquisas futuras, analisar outro grupo de empresas, bem como, países, a fim de verificar o grau de evidenciação ambiental de outros cenários, comparando com os resultados do presente estudo.

\section{REFERÊNCIAS BIBLIOGRÁFICAS}

[1] ALBERTINI, Elisabeth. A descriptive analysis of environmental disclosure: A longitudinal study of French companies. Journal of Business Ethics, v. 121, n. 2, p. 233-254, 2013.

[2] AL-TUWAIJRI, Sulaiman A.; CHRISTENSEN, Theodore E.; HUGHES II, K. E. The relations among environmental disclosure, environmental performance, and economic 
performance: a simultaneous equations approach. Accounting, Organizations and Society, v. 29, n. 5, p. 447-471, 2004.

[3] BEWLEY, Kathryn; LI, Yue. Disclosure of Environmental Information by Canadian Manufacturing Companies: A Voluntary Disclosure Perspective. Advances in Environmental Accounting \& Management, v. 1, n. 1, p. 201-226, 2000.

[4] BOFF, Marines Lucia. Estratégias de Legitimidade Organizacional de Lindblom na Evidenciação Ambiental e Social em Relatórios da Administração de Empresas Familiares. 2007. 160 f. Dissertação (Mestrado em Ciências Contábeis) - Programa de Pós-Graduação em Ciências Contábeis da Universidade Regional de Blumenau, Blumenau, 2007.

[5] BULGURCU, Berna Kiran. Application of TOPSIS technique for financial performance evaluation of technology firms in Istanbul stock exchange market. Procedia - Social and Behavioral Sciences, v. 62, n. 1, p. 1033-1040, 2012.

[6] BOWRIN, Anthony R. Corporate social and environmental reporting in the Caribbean. Social Responsibility Journal, v. 9, n. 2, p. 259-280, 2013.

[7] CLARKSON, Peter M.; LI, Yue; RICHARDSON, Gordon D.; VASVARI, Florin P. Revisiting the relation between environmental performance and environmental disclosure: An empirical analysis. Accounting, Organizations and Society, v. 33, n. 4, p. 303-327, 2008.

[8] CLARKSON, Peter M.; FANG, Xiaohua; LI, Yue; RICHARDSON, Gordon. The relevance of environmental disclosures: Are such disclosures incrementally informative?. Journal of Accounting and Public Policy, v. 32, n. 5, p. 410-431, 2013.

[9] ERTUGRUL, Irfan; KARAKASOGLU, Nilsen. Performance evaluation of Turkish cement firms with fuzzy analytic hierarchy process and TOPSIS methods. Expert Systems with Applications, v. 36, n. 1, p. 702-715, 2009.

[10] GRAY, Rob; JAVAD, Mohammed; POWER, David M.; SINCLAIR, C. Donald. Social and Environmental Disclosure and Corporate Characteristics: A Research Note and Extension. Journal of Business Finance \& Accounting, v. 28, n. 3-4, p. 327-356, 2001.

[11] GRI - Global Reporting Initiative. Princípios para Relato e Conteúdos Padrão. 2013.

[12] IATRIDIS, George Emmanuel. Environmental disclosure quality: Evidence on environmental performance, corporate governance and value relevance. Emerging Markets Review, v. 14, n. 1, p. 55-75, 2013.

[13] LU, Yingjun; ABEYSEKERA, Indra. Stakeholders' power, corporate characteristics, and social and environmental disclosure: evidence from China. Journal of Cleaner Production, v. 64, n. 1, p. 426-436, 2014.

[14] LYNCH, Barbara. An examination of environmental reporting by Australian state government departments. Accounting Forum, v. 34, n. 1, p. 32-45, 2010.

[15] PARK, Jeehye; BRORSON, Torbjörn. Experiences of and views on third-party assurance of corporate environmental and sustainability reports. Journal of Cleaner Production, v. 13, n. 10, p. 1095-1106, 2005.

[16] RAHAMAN, Abu Shiraz; LAWRENCE, Stewart; ROPER, Juliet. Social and environmental reporting at the VRA: institutionalised legitimacy or legitimation crisis?. Critical Perspectives on Accounting, v. 15, n. 1, p. 35-56, 2004. 
[17] ROSA, Fabrícia Silva da; FERREIRA, Araceli Cristina de Sousa; ENSSLIN, Sandra Rolim; ENSSLIN, Leonardo. Evidenciação Ambiental (EA): Contribuição da Metodologia Multicritério para Identificação dos Aspectos Financeiros para a Gestão Ambiental. Revista Contabilidade Vista \& Revista, v. 21, n. 4, p. 27-61, 2010.

[18] ROSA, Fabrícia Silva da; ENSSLIN, Sandra Rolim; ENSSLIN, Leonardo; LUNKES, Rogério João. Gestão da evidenciação ambiental: um estudo sobre as potencialidades e oportunidades do tema. Engenharia Sanitária Ambiental, v. 16, n. 1, p. 157-166, 2011.

[19] ROSA, Fabricia Silva da; GUESSER, Tatiana; HEIN, Nelson; PFITSCHER, Elisete Dahmer; LUNKES, Rogério João. Environmental impact management of Brazilian companies: analyzing factors that influence disclosure of waste, emissions, effluents, and other impacts. Journal of Cleaner Production, p. 1-13, 2013.

[20] ROSA, Fabricia Silva da; LUNKES, Rogério João, HEIN, Nelson; VOGT, Mara; DEGENHART, Larissa. Analysis of the determinants of disclosure of environmental impacts of Brazilian companies. Global Advanced Research Journal of Management and Business Studies, v. 3, n. 6, p. 249-266, 2014.

[21] TRIERWEILLER, Andréa Cristina; SEVERO PEIXE, Blênio César; BORNIA, Rafael Tezza, Antonio Cezar; CAMPOS, Lucila M.S. Measuring Environmental Management Disclosure in Industries in Brazil with item response Theory. Journal of Cleaner Production, v. 47, p. 298-305, 2012.

[22] TZENG, Gwo-Hshiung; HUANG, Jih-Jeng. Multiple Attribute Decision Making: Methods and Applications. CRC Press, 2011.

[23] WANG, Hua; BERNELL, David. Environmental Disclosure in China: An Examination of the Green Securities Policy. The Journal of Environment \& Development, v. 22, n. 4, p. 339-369, 2013.

[24] WU, Hung-Yi; TZENG, Gwo-Hshiung; CHEN, Yi-Hsuan. A fuzzy MCDM approach for evaluating banking performance based on Balanced Scorecard. Expert Systems with Applications, v. 36, n. 6, p. 10135-10147, 2009.

[25] ZELENY, Milan. Multiple Criteria Decision Making. McGraw-Hill: New York, 1982.

[26] ZENG, S. X.; XU, X.D.; YIN, H. T.; TAM, C. M. Factors that drive Chinese listed companies in voluntary disclosure of environmental information. Journal of Business Ethics, v. 109, n. 3, p. 309-321, 2012. 